\title{
HIF-1 $\alpha$ is a crucial factor in the development of peritoneal dissemination via natural metastatic routes in scirrhous gastric cancer
}

\author{
SHUUSUKE MIYAKE ${ }^{1}$, YOSHIHIKO KITAJIMA ${ }^{1,3}$, JUN NAKAMURA ${ }^{1}$, KEITA KAI ${ }^{2}$, \\ KAZUYOSHI YANAGIHARA ${ }^{4}$, TOMOKAZU TANAKA ${ }^{1}$, MASATSUGU HIRAKI ${ }^{1}$, \\ KOHJI MIYAZAKI ${ }^{5}$ and HIROKAZU NOSHIRO ${ }^{1}$
}

\author{
Departments of ${ }^{1}$ Surgery and ${ }^{2}$ Pathology and Biodefense, Saga University Faculty of Medicine, Saga 849-8501; \\ ${ }^{3}$ Department of Surgery, NHO Higashisaga Hospital, Miyaki-Town, Miyaki-Gun, Saga 849-0101; \\ ${ }^{4}$ Division of Genetics, National Cancer Center Research Institute, Chuo-ku, Tokyo 104-0045; \\ ${ }^{5}$ Saga University Faculty of Medicine, Saga 849-8501, Japan
}

Received May 27, 2013; Accepted July 2, 2013

DOI: 10.3892/ijo.2013.2068

\begin{abstract}
The molecular mechanisms underlying the peritoneal dissemination of gastric cancer remain unclear. Using in vivo metastatic models, this study attempted to clarify the role of hypoxia inducible factor (HIF)- $1 \alpha$ in the development of peritoneal dissemination of gastric cancer. HIF- $1 \alpha$ knockdown (KD) cells were established in the scirrhous gastric cancer cell line 58As9. Using KD and control (SC) cells, the presence of peritoneal dissemination was assessed in orthotopic implantation (o.i.) and intraperitoneal injection (i.p.) models. A series of in vitro analyses were also conducted. Finally, tumor angiogenesis was immunohistochemically analyzed. In the o.i. model, peritoneal dissemination was more frequently observed in the SC mice (93\%) compared to the KD mice $(13 \%)(\mathrm{P}<0.001)$. In the i.p. model, peritoneal dissemination occurred at a high rate in both types of mice; however, a greater number of nodules was observed in the $\mathrm{KD}$ mice $(\mathrm{P}=0.017)$. The in vitro assays showed that HIF-1 $\alpha$ exerts unfavorable effects on anoikis resistance and adhesion to extracellular matrix. Angiogenesis and vascular invasion were more aggressive in the SC gastric tumors. Vascular invasion was present in the intratumoral regions of the disseminated nodules in the SC o.i., but not the i.p., mice. HIF-1 $\alpha$ was found to be crucial for the development of peritoneal dissemination in o.i. model, which mimics natural metastasis. In contrast, HIF-1 $\alpha$ played an inhibitory role in suppressing peritoneal dissemination in the i.p. model. These
\end{abstract}

Correspondence to: Dr Yoshihiko Kitajima, Department of Surgery, Saga University Faculty of Medicine, 5-1-1 Nabeshima, Saga 849-8501, Japan

E-mail: kitajiy@esaga.hosp.go.jp

Key words: gastric cancer, peritoneal dissemination, HIF-1 $\alpha$, angiogenesis results indicate that peritoneal dissemination in o.i. mice may not act through a seeding mechanism. An immunohistochemical analysis demonstrated HIF- $1 \alpha$-activated angiogenesis and vascular invasion in stomach tumors. Furthermore, the results showed that the disseminated nodules observed in SC o.i. mice were formed via extravasation of cancer cells. We provide a possible mechanism in which peritoneal dissemination of gastric cancer develops via a vascular network whereby HIF-1 $\alpha$ activates tumor angiogenesis.

\section{Introduction}

Gastric cancer is the fourth most common cancer and the second leading cause of cancer-related death (1). Peritoneal dissemination is one of the terminal features of gastric cancer leading to death. There is currently no standard treatment for peritoneal dissemination, as neither surgery nor chemotherapy exert beneficial effects on survival (2). Therefore, investigating the molecular mechanisms underlying peritoneal dissemination is required in order to improve the clinical outcomes of gastric cancer.

Peritoneal dissemination is currently believed to develop via a direct seeding mechanism. The seeding theory is composed of several sequential steps, including cancer invasion in the gastric wall, detachment of cancer cells from the primary tumor, attachment to the distant peritoneum, invasion into the subperitoneal space and proliferation with vascular neogenesis (3). However, most proposed theories remain speculative and are seldom based on adequate evidence. The intraperitoneal injection (i.p.) of cultured gastric cancer cells into the peritoneal cavity in nude mice is currently used as a principle model to mimic the development of peritoneal dissemination (4). This model actually disregards the initial steps of the seeding theory, including cancer invasion in the gastric wall and detachment of cancer cells from the primary tumor. Therefore, it is difficult to conclude that the i.p. model accurately reflects the characteristics of peritoneal dissemination originating from primary gastric cancer. 
On the other hand, three scirrhous gastric cancer cell lines (44As3, 58As1 and 58As9) have been established by repeating orthotopic implantation (o.i.) in nude mice (5). When these cells are implanted into the stomach wall in nude mice, dissemination to the greater omentum and mesentery and the formation of bloody ascites are frequently (90-100\%) observed (5). Therefore, the o.i. model using these cells is a powerful tool for analyzing the mechanisms underlying the spread of peritoneal dissemination via natural metastatic routes. Recently, one study examined this natural metastasis model and reported the essential role of miR-516a-3p in the development of peritoneal dissemination (6).

The transcription factor hypoxia-inducible factor (HIF)- $1 \alpha$ is stabilized under hypoxic conditions and plays an essential role in oxygen homeostasis (7). Various genes that regulate energy metabolism, angiogenesis, apoptosis and cell survival have been identified to be HIF-1 $\alpha$ targets (8-10). Furthermore, previous reports have revealed that hypoxia and HIF-1 $\alpha$ overexpression increase tumor aggressiveness and chemoresistance in various cancers, including gastric cancer (11-24). Recent studies have shown that each step of cancer metastasis, from the initial epithelial-mesenchymal transition to the final step of organotropic colonization, is potentially regulated by hypoxia, suggesting that HIF-1 $\alpha$ plays a role as a master regulator in cancer metastasis (24). Although numerous in vitro experiments have addressed the importance of HIF-1 $\alpha$ in cancer metastasis, in vivo studies have not been well documented.

In the present study, we newly established knockdown (KD) cells using scirrhous gastric cancer 58As9 cells in which the HIF-1 $\alpha$ expression was stably knocked down via siRNA transfection. Then, we investigated the metastatic potential for peritoneal dissemination in the $\mathrm{KD}$ cells and control transfectant (SC) cells using orthotopic implantation (o.i.) and intraperitoneal injection (i.p.) in nude mice. Finally, the presence of tumor angiogenesis in the primary stomach tumors and the disseminated mesenteric nodules was compared between the KD and SC mice. The goal of this study was to clarify whether the HIF-1 $\alpha$ expression is required for the development of peritoneal dissemination and to elucidate whether this type of metastasis can be achieved via a direct seeding mechanism.

\section{Materials and methods}

Cell culture and exposure to hypoxia. The gastric cancer cell line 58As9 (5) was kindly provided by Dr K. Yanagihara (National Cancer Research Institute, Tokyo, Japan). The cells were cultured in RPMI-1640 medium (Sigma-Aldrich, Inc., St. Louis, MO, USA) and maintained under conditions of either normoxia $\left(20 \% \mathrm{O}_{2}\right.$ and $5 \% \mathrm{CO}_{2}$ in air) or hypoxia $\left(1 \% \mathrm{O}_{2}, 5 \% \mathrm{CO}_{2}\right.$ and $\left.94 \% \mathrm{~N}_{2}\right)$.

HIF-1 $\alpha$ RNA interference. The pBAsi-hU6 Pur DNA plasmid vector (Takara Biotechnology, Shiga, Japan) was used to construct a HIF-1 $\alpha$ siRNA plasmid by inserting an siRNAcoding sequence under the U6 promoter. The sequences of siRNA targeting HIF- $1 \alpha$ and control scrambled siRNA were designed as follows: HIF-1 $\alpha$ (5'-CCA CAT TCA CGT ATA TGA T-3') and scrambled (5'-TCT TAA TCG CGT ATA AGG C-3'). The 58As9 cells were transfected using a MicroPorator-mini (MP-100) (Digital Bio Technology, Seoul,
Korea) according to the manufacturer's instructions. To obtain KD cells and control SC cells with stable transfection of the above sequences, the cells were selected using puromycin (Sigma) at a concentration of $3.0 \mu \mathrm{g} / \mathrm{ml}$, then maintained in complete medium supplemented with puromycin.

Western blot analysis. Whole cell lysates obtained from the cultured cells were prepared using a lysis buffer and protease inhibitor cocktail mix (Roche, Mannheim, Germany). For the western blot analysis, the samples were dissolved in NuPage ${ }^{\mathrm{TM}}$ LDS sample buffer (Invitrogen Corp., Carlsbad, CA, USA) and $1 \mathrm{M}$ dithiothreitol, then heated for $5 \mathrm{~min}$ at $95^{\circ} \mathrm{C}$. Aliquots containing $30 \mu \mathrm{g}$ of protein were subjected to $4-12 \%$ Bis-Tris Gel (Invitrogen) and electrophoretically transferred onto an Amersham $^{\mathrm{TM}}$ Hybond $^{\mathrm{TM}}$-ECL membrane (GE Healthcare, Buckinghamshire, UK) in a transfer buffer. After blocking with $5 \%$ skim milk for $30 \mathrm{~min}$, the membrane was incubated with primary antibodies overnight at $4^{\circ} \mathrm{C}$. The primary antibodies used for the western blot analyses were anti-HIF-1 $\alpha$ (1:1000, Epitomics, Burlingame, CA, USA) and anti- $\beta$-actin (1:10,000, Sigma) antibodies. Following incubation with the corresponding secondary antibodies, the signals were developed using the Amersham ${ }^{\mathrm{TM}}$ ECL Plus Western Blotting Detection System (GE Healthcare).

Cell viability assay. The cell viability was analyzed using an MTT assay with a Cell-Titer $96^{\mathrm{TM}}$ non-radioactive cell proliferation assay kit (Promega, Madison, WI, USA). In brief, $5 \times 10^{3}$ cells per well were seeded in triplicate onto 96 -well plates and incubated under normoxia and hypoxia at $37^{\circ} \mathrm{C}$ in a humidified atmosphere. After $24 \mathrm{~h}$, the number of viable cells was measured in triplicate every day for three days. Cell viability curves were then constructed by calculating the mean values of the optical density measurements at $590 \mathrm{~nm}$ using a 96-well plate reader (Immuno-mini NJ2300, Nalge Nunc International K.K., Tokyo, Japan).

In vitro invasion assay. Polycarbonate filters (6.5-mm-diameter) $\left(8-\mu \mathrm{m}\right.$ pore size) of Falcon Transwell ${ }^{\mathrm{TM}}$ chemotaxis chambers (Beckton-Dickinson, Franklin Lakes, NJ, USA) were coated with $50 \mu \mathrm{l}(1 \mathrm{mg} / \mathrm{ml})$ of Matrigel biomatrix (Beckton-Dickinson) in cold RPMI-1640 medium and dried overnight. Suspensions of $1 \times 10^{5}$ cells in $200 \mu \mathrm{l}$ of complete RPMI-1640 medium were placed in the upper compartments of the chamber, while the lower compartments were filled with $800 \mu \mathrm{l}$ of conditioned medium obtained from MRC5 fibroblasts. The culture units were incubated for $24 \mathrm{~h}$ at $37^{\circ} \mathrm{C}$ under normoxia and hypoxia. The non-invasive cells on the upper surface of the filters were then completely removed using a cotton swab. Any viable invasive cells that had infiltrated onto the lower surface of the filter were fixed with $70 \%$ ethanol and the nuclei were stained with hematoxylin. Next, the number of invasive cells was counted. The experiments were performed in triplicate and independently repeated at least three times.

Animal experiments. The animal experimental protocols were approved by the Animal Care Committee of Saga University. The mice were purchased from CLEA Japan (Tokyo, Japan) and maintained under specific pathogen-free conditions. The animals were provided sterile food and water and housed in 
cages. The ambient light was controlled to provide regular 12-h light-dark cycles.

Orthotopic implantation. Six-week-old female BALB/c nude mice were anesthetized using intraperitoneal injections of 2.2.2-tribromoethanol (Aldrich Chemical, Milwaukee, WI, USA) at a dose of $0.28 \mathrm{mg} / \mathrm{g}$ body weight. In each mouse, a small median abdominal incision was made under anesthesia and $2 \times 10^{6}$ cells in a 50- $\mu 1$ volume of RPMI medium were then inoculated into the middle wall of the greater curvature of the stomach using a 30-gauge needle (Nipro, Tokyo, Japan). Each of the KD and SC cell lines were orthotopically implanted into 15 mice. The mice were sacrificed 70 days after tumor cell inoculation or when they became moribund and the degree of peritoneal dissemination was evaluated by counting the number of tumor nodules on the mesentery. In each case, the stomach and mesenteric tumors were processed for the histological examinations.

Intraperitoneal injection method used in the peritoneal dissemination model. The i.p. method used in the peritoneal dissemination model was performed according to the procedures described in a previous report with slight modifications (25). To generate the xenograft model, cancer cells $\left(2 \times 10^{6}\right)$ were suspended in $200 \mu \mathrm{l}$ of PBS and injected on day 0 into the abdominal cavity. Seven mice per group were injected with each cell line. All of the mice were sacrificed on day 21 and the number of disseminated nodules was counted.

Anchorage-independent cell viability assay. The anchorageindependent cell viability was assessed using the MTT method described in the cell viability assay section. The assay was performed using 96-well plates coated with Ultra-Low Attachment Surface (Corning, NY, USA) instead of normal plates without any coating.

Adhesion assay. To quantify the number of tumor cells attached to the extracellular matrix (ECM), an in vitro adhesion assay was performed using a CytoSelect 48-well cell adhesion assay (ECM array, Colorimetric) (Cell Biolabs, Inc., San Diego, CA, USA) according to the manufacturer's instructions. Regarding the analysis performed under hypoxic conditions, the cells were incubated for $24 \mathrm{~h}$ at $37^{\circ} \mathrm{C}$ under hypoxia before the assay was performed.

Immunohistochemistry. The paraffin-embedded samples were cut into 4- $\mu \mathrm{m}$-thick sections, then deparaffinized in xylene and rehydrated in a graded series of ethanol. For antigen retrieval, the slides were heated in EDTA (a pH of 8.0 for HIF-1 $\alpha$ and $\mathrm{Ki}-67$ and a $\mathrm{pH}$ of 9.0 for LYVE-1) in a microwave for 6 min or incubated with proteinase $\mathrm{K}$ for $10 \mathrm{~min}$ (for PECAM-1) at room temperature. The following primary antibodies were used: mouse monoclonal anti-human HIF-1 $\alpha$ (1:50, clone HI-67; Novus Biologicals, Littleton, CO, USA), mouse monoclonal anti-human Ki-67 (1:30, clone MIB-1; Dako Cytomation, Glostrup, Denmark), rabbit polyclonal anti-mouse LYVE-1 (1:30, 103-PA50AG; RELIA Tech GmbH, Wolfenb üttel, Germany) and platelet endothelial cell adhesion molecule 1 (PECAM-1) (1:50, clone MEC13.3; Santa Cruz Biotechnology, Santa Cruz, CA, USA). The Envision ${ }^{+}$System (Dako
Cytomation) was used as the secondary antibody for HIF-1 $\alpha$, Ki-67 and LYVE-1. Biotinylated rabbit anti-rat (E0468; Dako Cytomation) secondary antibodies were used for PECAM-1. Immunoreactive proteins were detected using an avidinbiotin-based peroxidase system or peroxidase streptavidin (Nichirei Co., Tokyo, Japan). The signals were visualized with diaminobenzidine tetrahydrochloride $(0.02 \%)$. The cell nuclei were counterstained with Mayer's hematoxylin (Merck KGaA, Darmstadt, Germany). The immunohistochemical expressions of HIF-1 $\alpha$, Ki-67, PECAM-1 and LYVE-1 were assessed by a certified pathologist (K. Kai).

Microvessel density assessment. The areas with larger numbers of microvessels in the tissue sections were marked under microscopy. Using a magnification of $\mathrm{x} 400$, three optical fields were selected. All single stained endothelial cells or cell clusters clearly separated from the adjacent microvessels were considered to be single countable microvessels. The microvessel density (MVD) was defined as the mean number of microvessels per optical field (among the three selected optical fields).

Assessment of angiogenesis and lymphangiogenesis. The blood vessels were visualized using immunohistochemical staining of PECAM-1 and assessed according to the MVD of PECAM-1-positive vessels. Lymphangiogenesis was evaluated both by counting the number and measuring the diameter of the LYVE-1-positive vessels in which cancer cells had infiltrated. The mean number and mean diameter were calculated in 10 of the SC and seven of the KD stomach tumors.

Total RNA extraction and real-time quantitative reverse transcription-polymerase chain reaction. Total RNA was extracted from each cell line using an Isogen ${ }^{\circledR}$ RNA extraction kit (Nippon Gene, Osaka, Japan). For each cell line, $1 \mu \mathrm{g}$ of RNA was converted into cDNA using a ReverTra Ace (Toyobo) reverse transcription reaction kit. The cDNA was used as a template for the PCR. Real-time RT-PCR was performed by means of the LightCycler ${ }^{\mathrm{TM}}$ instrument system (Roche) using the Light-Cycler-FastStart DNA Master ${ }^{\mathrm{TM}}$ SYBR Green I kit (Roche). The primers were designed as follows: vascular endothelial growth factor (VEGF)-A: (5'-GCA GAA TCA TCA CGA AGT GG-3', 5'-GCA TGG TGA TGT TGG ACT CC-3', product length $212 \mathrm{bp}$ ), aldolase C (ALDOC): (5'-AAA TTG GGG TGG AAA ACA CA-3', 5'-AGA AAA TGA CGC CTC CAA TG-3', 102 bp), carbonic anhydrase (CA) 9: (5'-CCG AGC GAC GCA GCC TTT GA-3', 5'-GGC TCC AGT CTC GGC TAC CT-3', 252 bp) and $\beta$-actin: (5'-ACT CTT CCA GCC TTC CTT CC-3', 5'-GAC AGC ACT GTG TTG GCG TA-3', 120 bp). After performing a denaturation step at $95^{\circ} \mathrm{C}$ for $3 \mathrm{~min}$, PCR amplification was conducted with 50 cycles of $15 \mathrm{sec}$ of denaturation at $95^{\circ} \mathrm{C}, 5 \mathrm{sec}$ of annealing at $60^{\circ} \mathrm{C}$ and $10 \mathrm{sec}$ of extension at $72^{\circ} \mathrm{C}$. The quantitative values were normalized to the $\beta$-actin expression. All experiments were performed in triplicate and the mean values were calculated.

Statistical analysis. The statistical analysis was performed using the computer software program SPSS 19.0 for Mac (SPSS, Chicago, IL, USA). Comparisons between two groups were made using Student's t-test and the Mann Whitney U test. 

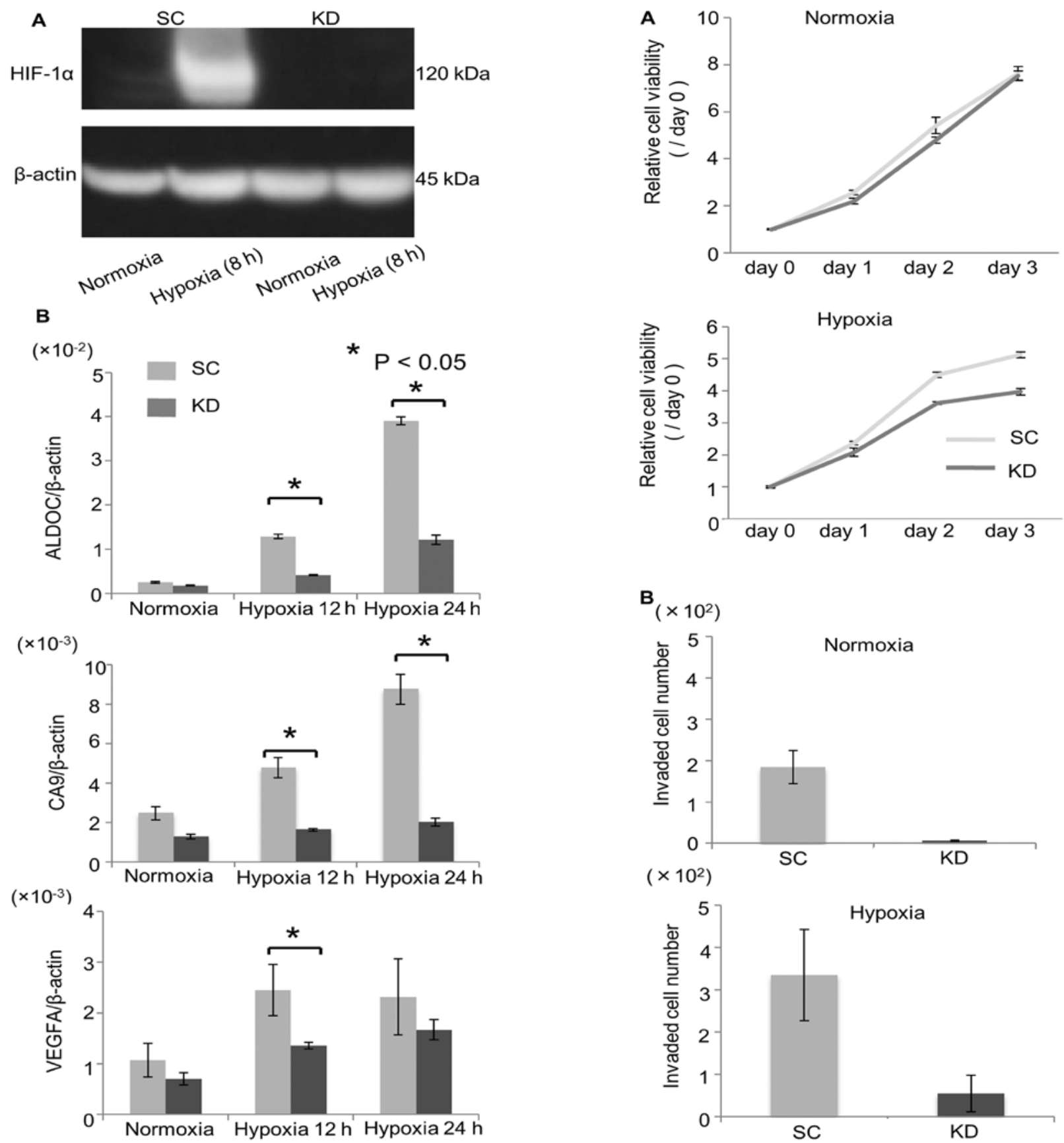

Figure 1. Expressions of HIF-1 $\alpha$ and its target genes in the KD and SC cells. (A) Western blot analysis of HIF-1 $\alpha$ in the KD cells, in which the HIF-1 $\alpha$ expression was stably knocked down by transfection of siRNA plasmids and the corresponding control transfectant SC cells. The HIF-1 $\alpha$ expression was induced by hypoxia in the SC, but not KD, cells. (B) Quantitative RT-PCR of the HIF-1 $\alpha$ target genes. HIF-1 $\alpha$ knockdown in the KD cells resulted in a greater suppression of the hypoxic induction of HIF-1 $\alpha$ target genes, such as ALDOC, CA9 and VEGF-A, compared with that observed in the SC cells.

Survival curves were generated according to the Kaplan-Meier method and statistical differences were compared using the log-rank test. P-values of $<0.05$ were considered to be statistically significant.

\section{Results}

$H I F-1 \alpha$ siRNA significantly decreases the expression of $H I F-1 \alpha$ and its target genes. The HIF-1 $\alpha$ expression in the

SC and KD cells was validated using a western blot analysis (Fig. 1A). In the SC cells, HIF-1 $\alpha$ was faintly expressed under normoxia, while the HIF-1 $\alpha$ expression was strongly induced

Figure 2. Assessment of the viability and invasive ability of KD and SC cells. (A) MTT cell viability assays were performed under normoxic and hypoxic conditions. The MTT activity was measured in triplicate on days $0,1,2$ and 3 . Cell viability curves were created by plotting the mean values of the optical densities measured at $590 \mathrm{~nm}$ using a 96 -well plate reader. The relative viability values (mean $\pm \mathrm{SD}$ ) on days 1-3 are shown as ratios to the value on day 0 . There were no significant differences in the relative proliferation values on day 3 between the KD and SC cells under normoxic conditions. In contrast, the KD cells exhibited a significantly lower level of viability than the SC cells under hypoxic conditions $(5.12 \pm 0.09$ vs. $3.97 \pm 0.10, \mathrm{P}<0.001)$. (B) The invasive ability of the KD and SC cells was assessed using two-chamber Matrigel assays. The data are presented as the mean \pm SD of the triplicate measurements. The KD cells exhibited significantly less invasiveness than the SC cells under both normoxic and hypoxic conditions (normoxia: $1.84 \times 10^{2} \pm 48.8$ vs. $0.06 \times 10^{2} \pm 2.08, \mathrm{P}=0.003$; hypoxia: $3.35 \times 10^{2} \pm 1.32 \times 10^{2}$ vs. $\left.0.55 \times 10^{2} \pm 53.1, \mathrm{P}=0.027\right)$. 
A
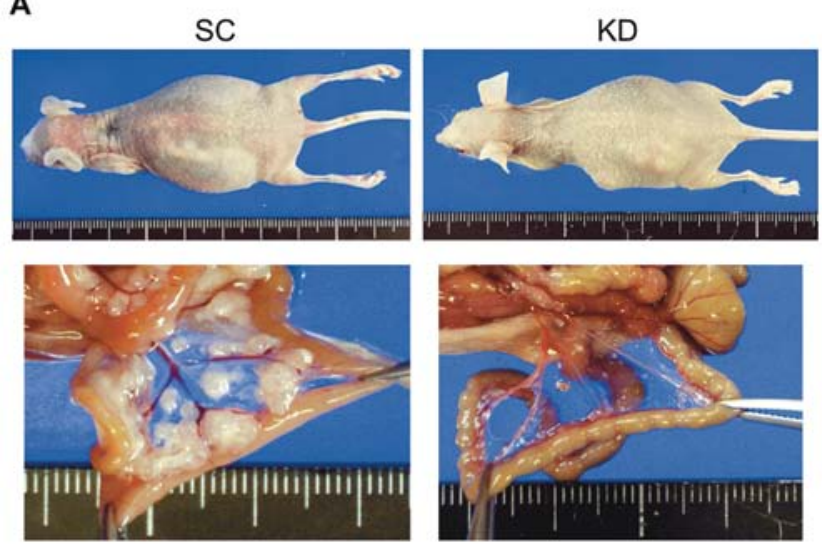

C

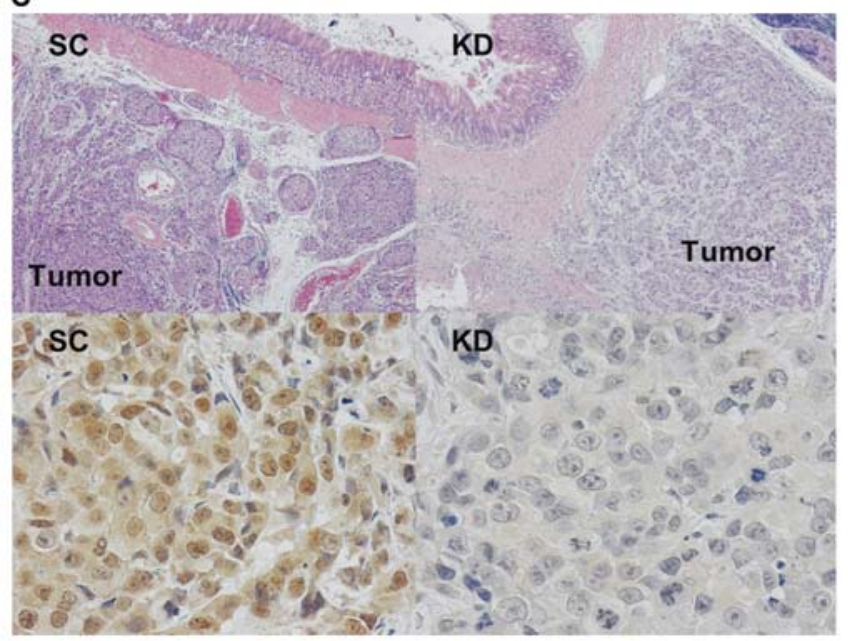

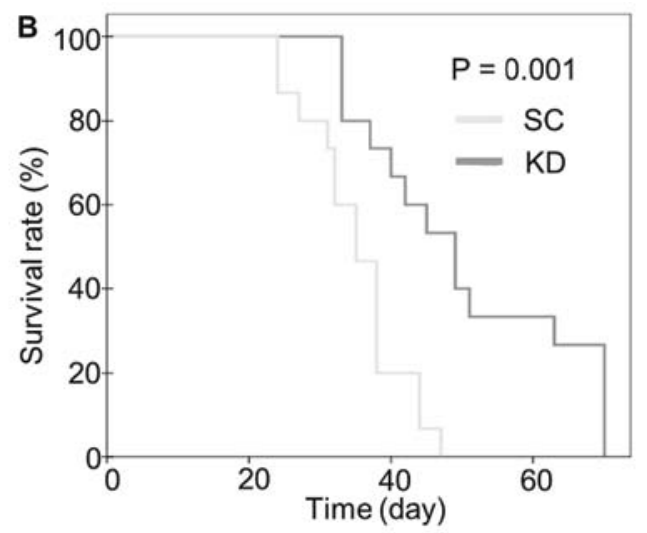

D

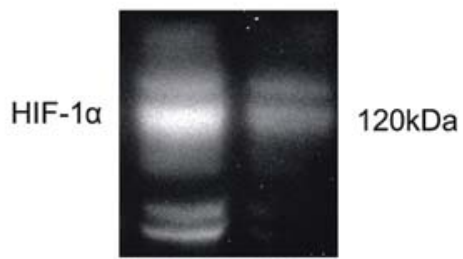

$\beta$-actin

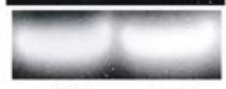

$45 \mathrm{kDa}$

$\mathrm{SC} \quad \mathrm{KD}$

E

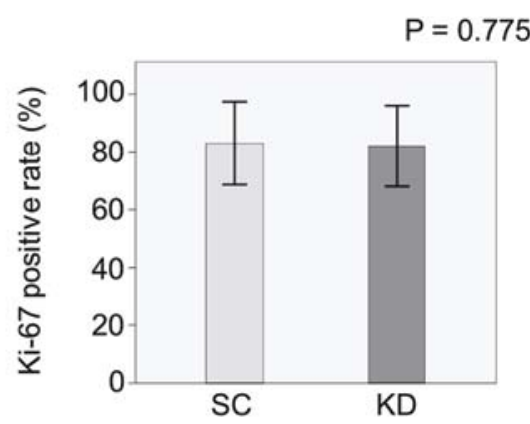

Figure 3. Assessment of peritoneal dissemination, survival and implanted stomach tumors in the o.i. mice. (A) Macroscopic appearance of the nude mice that underwent o.i. of KD and SC cells. Abdominal distension due to bloody ascites was evident in the SC, but not KD, mice (upper panel). Multiple nodules of peritoneal dissemination were observed on the mesentery in the SC, but not KD, mice. (B) Comparison of survival between the KD and SC mice. The survival of the KD mice was significantly longer than that of the SC mice $(n=15 ; P=0.001)$. (C) Microscopic findings of the orthotopically implanted xenografts in the murine stomachs. Hematoxylin and eosin staining (x100) (upper panel). Both SC and KD cells grew under the mucosal layer. Immunohistochemical staining of HIF-1 $\alpha$ using the tumor sections (x400) (lower panel). Positive staining of HIF-1 $\alpha$ was diffusely observed in the nuclei of the SC, but not KD, cells. (D) Western blot analysis of the HIF-1 $\alpha$ expression in the stomach tumors. The protein expression of HIF-1 $\alpha$ was lower in the tumors derived from the KD cells than in the $\mathrm{SC}$ cells. (E) Comparison of cell proliferation in the stomach tumors estimated using Ki-67 staining. There were no significant differences in the rate of Ki-67 positivity between the $\mathrm{SC}$ and $\mathrm{KD}$ cells $(\mathrm{P}=0.775)$.

by hypoxia. On the other hand, hypoxic induction of the HIF-1 $\alpha$ expression was strongly reduced in the KD cells. To assess the knockdown effects of HIF-1 $\alpha$, an RT-PCR analysis was performed on HIF-1 $\alpha$ target genes, such as aldolase $\mathrm{C}$ (ALDOC), carbonic anhydrase (CA) 9 and vascular endothelial growth factor (VEGF)-A. The hypoxic induction of these genes was significantly reduced in the KD cells compared with that observed in the SC cells (Fig. 1B).

Effects of HIF-1a knockdown on cell viability and invasion. The cell viability and invasion activity were compared between the KD and SC cells. As shown in Fig. 2A, the cell viability did not differ between the KD and SC cells under normoxia, whereas the cell viability under hypoxia was significantly decreased in the KD cells. The invasive ability of the KD cells was strongly decreased compared with that observed in the SC cells under both normoxia and hypoxia (Fig. 2B).
Comparison of the KD and SC cells in the orthotopic implantation model. Orthotopic implantation of SC cells resulted in the development of massive ascites in the mice 20 days after injection and several mice became moribund (Fig. 3A). In contrast, o.i. of KD cells, resulted in moribundity without ascites formation $\sim 33$ days after injection. Furthermore, the KD mice demonstrated significantly longer survival times than the SC mice (Fig. 3B). When the dead animals were autopsied, the primary stomach tumors were highly developed in both the SC (15/15:100\%) and KD mice (13/15:86.7\%) (Table I). Peritoneal disseminated nodules with bloody ascites most often developed on the mesentery (Fig. 3A). The nodules were more frequently observed in the SC mice (14/15: 93.3\%) than in the KD mice (2/15: 13.3\%, P<0.001). (Fig. 3A, Table I). Fig. 3C demonstrates a series of microscopic analyses using $\mathrm{KD}$ and SC stomach tumors. Both the KD and SC stomach tumors grew beneath the mucosal layer. In the immunohistochemical 
Table I. Incidence of peritoneal dissemination following orthotopic implantation (o.i.) of SC and KD cells.

\begin{tabular}{|c|c|c|c|c|}
\hline $\begin{array}{l}\text { Cell } \\
\text { lines }\end{array}$ & $\begin{array}{c}\text { Survival } \\
\text { (days) }\end{array}$ & $\begin{array}{l}\text { Stomach } \\
\text { tumor }\end{array}$ & Ascites & $\begin{array}{c}\text { Peritoneal } \\
\text { dissemination }\end{array}$ \\
\hline $\mathrm{SC}$ & $\begin{array}{c}35 \pm 7^{\mathrm{a}} \\
(24-47)\end{array}$ & $\begin{array}{c}15 / 15 \\
(100 \%)\end{array}$ & $\begin{array}{c}14 / 15^{\mathrm{a}} \\
(93.3 \%)\end{array}$ & $\begin{array}{c}14 / 15^{\mathrm{a}} \\
(93.3 \%)\end{array}$ \\
\hline $\mathrm{KD}$ & $\begin{array}{c}50 \pm 15 \\
(33-70)\end{array}$ & $\begin{array}{c}13 / 15 \\
(86.7 \%)\end{array}$ & $\begin{array}{r}3 / 15 \\
(20 \%)\end{array}$ & $\begin{array}{c}2 / 15 \\
(13.3 \%)\end{array}$ \\
\hline
\end{tabular}

${ }^{\text {a }} \mathrm{P}<0.001$.

Table II. Incidence of peritoneal dissemination following intraperitoneal injection (i.p.) of SC and KD cells.

\begin{tabular}{lcc}
\hline Cell lines & Ascites & Peritoneal dissemination \\
\hline SC & $5 / 7$ & $7 / 7$ \\
& $(71.4 \%)$ & $(100 \%)$ \\
KD & $6 / 7$ & $7 / 7$ \\
& $(85.7 \%)$ & $(100 \%)$ \\
\hline
\end{tabular}

examination of HIF- $1 \alpha$, nuclear staining of this protein was observed in the SC, but not in the $\mathrm{KD}$, tumors. A western blot analysis also confirmed a higher expression of HIF-1 $\alpha$ in the $\mathrm{SC}$ tumors than in the KD tumors (Fig. 3D). In contrast, the percentage of cells exhibiting Ki-67 staining was almost the same between the KD and SC tumors (Fig. 3E; P=0.775).

Comparison of the $K D$ and SC cells in the intraperitoneal injection model. The results of i.p. in KD and SC cells were compared and with those observed in the o.i. model. Distinct from the o.i. model, peritoneal dissemination and ascites were frequently observed in both the KD i.p. and SC i.p. mice (Table II). Notably, the KD i.p. mice exhibited significantly greater numbers of disseminated nodules of smaller sizes than those observed in the SC i.p. mice (Fig. 4; $\mathrm{P}=0.017$ ).

Anchorage-independent cell viability and adhesion to the extracellular matrix. In vitro anchorage-independent cell viability assays and adhesion assays were performed to investigate why the KD cells developed greater numbers of peritoneal nodules than the SC cells in the i.p. model. The results showed that the anchorage-independent cell viability was higher in the KD cells under both normoxia and hypoxia (Fig. 5A). In the adhesion assay, the attachment value to fibronectin was significantly higher in the KD cells than in the SC cells under normoxic conditions (Fig. 5B; $\mathrm{P}=0.003$ ). However, the attachment values to collagen I, IV and laminin under hypoxia were significantly higher in the KD cells than in the SC cells (Fig. 5B; $\mathrm{P}<0.001, \mathrm{P}=0.003, \mathrm{P}=0.001$, respectively).

Immunohistochemistry for LYVE-1 and PECAM-1. Tumor angiogenesis was assessed not only in the implanted stomach tumors, but also in the disseminated nodules. Immunohistochemical staining of PECAM-1 as a blood vessel marker and LYVE-1 as a lymphatic vessel marker was assessed in the stomach tumors injected with KD or SC cells (Fig. 6). Vessels with a PECAM-1-positive expression were frequently observed at the tumor periphery in the SC stomach tumors (Fig. 6A), while such vessels were observed less frequently in the KD tumors and their tube formation was more immature (Fig. 6B). The mean MVD of the PECAM-1-positive vessels in the SC tumors was significantly higher than that observed in the KD tumors $(20.2 \pm 8.0$ vs $5.6 \pm 2.4, \mathrm{P}=0.001$, Fig. $6 \mathrm{E})$. LYVE-1 was also expressed at the tumor periphery in the SC tumors (Fig. 6C). Furthermore, vascular invasion, in which cancer cells invaded and grew inside the vessels, was frequently observed in the SC tumors. These aspects of lymphatic vessels were also found in the KD tumors; however, the tube formation appeared to be immature compared with that observed in the SC tumors (Fig. 6D). Indeed, the number as well as the diameter of the LYVE-1-positive vessels in the KD tumors were significantly smaller than those of the vessels observed in the $\mathrm{SC}$ tumors (Fig. $6 \mathrm{E} ; \mathrm{P}=0.043$ and $\mathrm{P}=0.04$ ). The presence of tumor angiogenesis in the disseminated mesenteric nodules
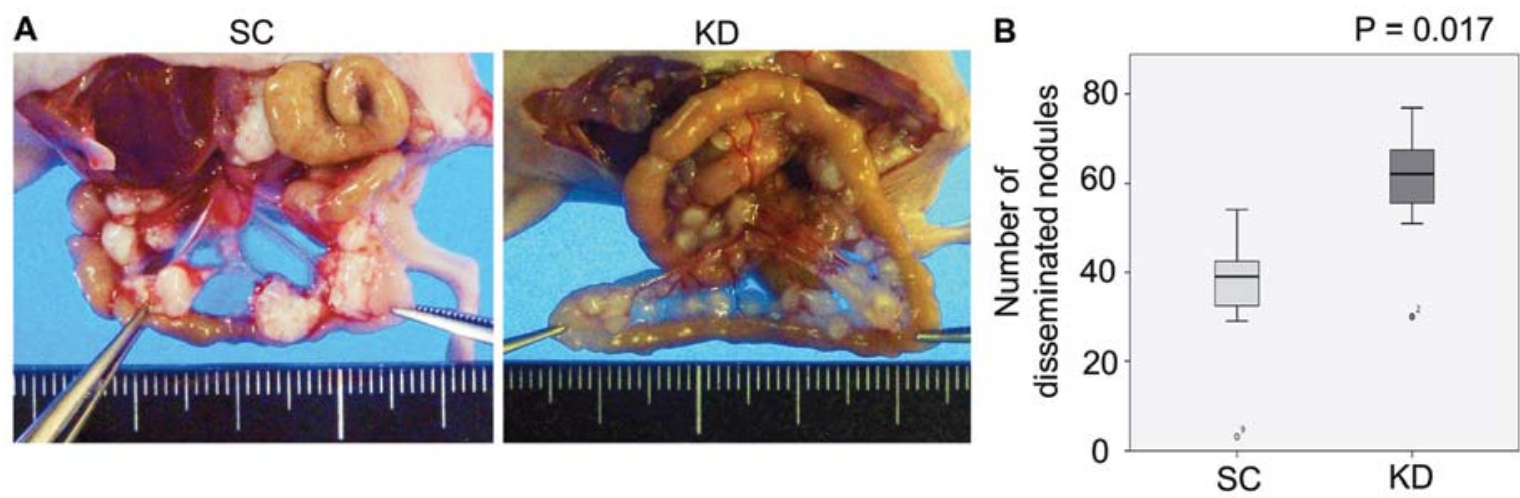

Figure 4. Assessment of disseminated nodules in the i.p. mice. (A) Macroscopic appearance of the nude mice that underwent i.p. of KD and SC cells. Disseminated nodules with a white color were observed on the mesentery in the KD and SC mice. The size of the nodules was larger in the SC mice than in the KD mice. (B) The total number of disseminated nodules was estimated in both the KD and SC i.p. mice (n=7). A significantly larger number of nodules was observed in the KD mice than in the SC mice $(\mathrm{P}=0.017)$. 

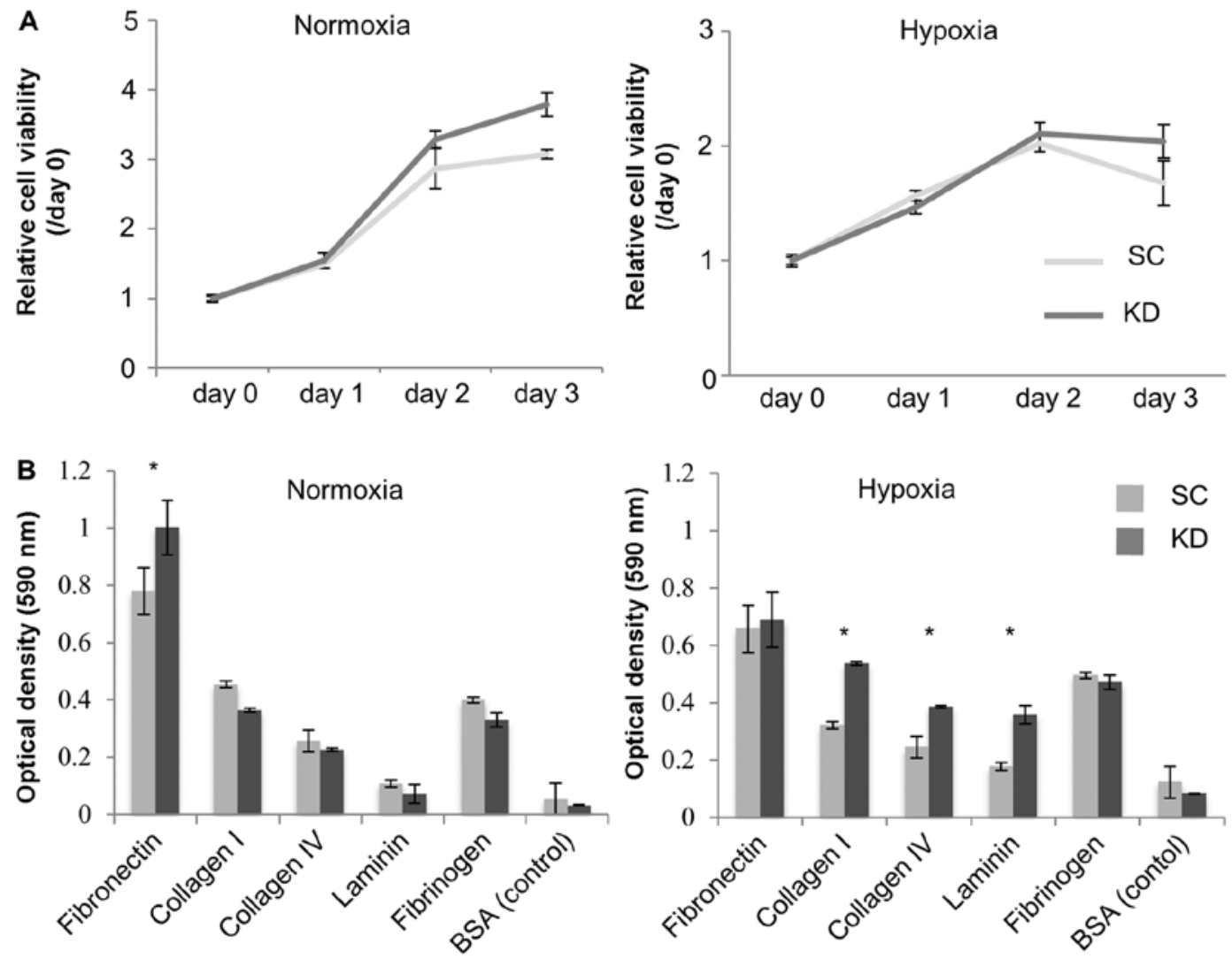

Figure 5. Assessment of anchorage-independent proliferation and adhesion ability in the KD and SC cells. (A) Anchorage-independent proliferation was assessed under normoxic and hypoxic conditions. The KD cells exhibited a significantly higher proliferative potential than the control cells on day 3 under both normoxic and hypoxic conditions (normoxia: $3.79 \pm 0.17$ vs. $3.07 \pm 0.06, \mathrm{P}<0.001$; hypoxia: $2.04 \pm 0.15$ vs. $1.68 \pm 0.19$, $\mathrm{P}=0.004$ ). (B) Results of the adhesion assays. Under the normoxic conditions, attachment to fibronectin was significantly decreased in the SC cells compared to that observed in the KD cells. Under the hypoxic conditions, attachment to collagen I, collagen IV and laminin was significantly increased in the KD cells compared to that observed in the SC cells. Attachment to fibronectin and fibrinogen under the hypoxic conditions was not significantly different between the KD and SC cells. ${ }^{*} \mathrm{P}<0.05$.

was further assessed (Fig. 7). In the nodules obtained from the SC o.i. mice, PECAM-1- and LYVE-1-positive vessels with cancer invasion were observed in the intratumoral regions (Fig. 7A and D). In sharp contrast, vessels lacking tumor invasion had formed at the tumor periphery in the nodules obtained from the SC i.p. and KD i.p. mice (Fig. 7B, C, E and F).

\section{Discussion}

The present study primarily aimed to investigate whether HIF-1 $\alpha$ is involved in the development of peritoneal dissemination using nude mouse models. First, stable transfectant KD cells lacking an HIF-1 $\alpha$ expression and control SC cells were established from parental 58As9 cells. A series of in vitro analyses confirmed the effective knockdown of HIF-1 $\alpha$ and the subsequent suppression of HIF-1 $\alpha$ target genes in the KD cells. A functional analysis revealed a significant reduction in the viability and invasive ability of the KD cells under hypoxia. In the o.i. model using KD and SC cells, primary tumors were frequently observed in the stomach wall in both types of mice, with no significant differences in the incidence of tumors. Furthermore, the degree of cell proliferation, as estimated based on Ki-67 immunostaining, did not differ between the tumors. With respect to the incidence of peritoneal dissemination and ascites production, significant differences were observed between the KD and SC o.i. mice. These results indicate that the loss of the HIF-1 $\alpha$ expression did not affect cell proliferation in the primary tumors, but rather strongly inhibited peritoneal dissemination and yielded worse survival outcomes in the SC o.i. mice.

In addition to the o.i. model, we further employed an i.p. model using these cells and analyzed the presence of disseminated nodules on the mesentery. The results demonstrated that peritoneal dissemination, as well as ascites production, frequently occurs in both KD i.p. and SC i.p. mice. Of interest, a greater number of disseminated nodules grew on the mesentery in the KD i.p. mice compared with that observed in the SC i.p. mice, although the nodules were smaller in size. We previously reported similar findings showing that the inactivation of HIF-1 $\alpha$ in the gastric cancer cells MKN45 and MKN74 induces the formation of a greater number of mesenteric nodules in the i.p. model (25). Additionally, in an in vitro analysis, higher resistance against anoikis was observed in the KD cells under both normoxic and hypoxic conditions. Regarding the adhesion ability of cells to components of the extracellular matrix, such as laminin and collagen I and IV, a stronger activity was observed in the KD cells than in the SC cells under hypoxia. Laminin and collagen I and IV are major components of the mesothelial basement membrane and play key roles in the initial adhesion of cancer cells to the peritoneum $(26,27)$. 

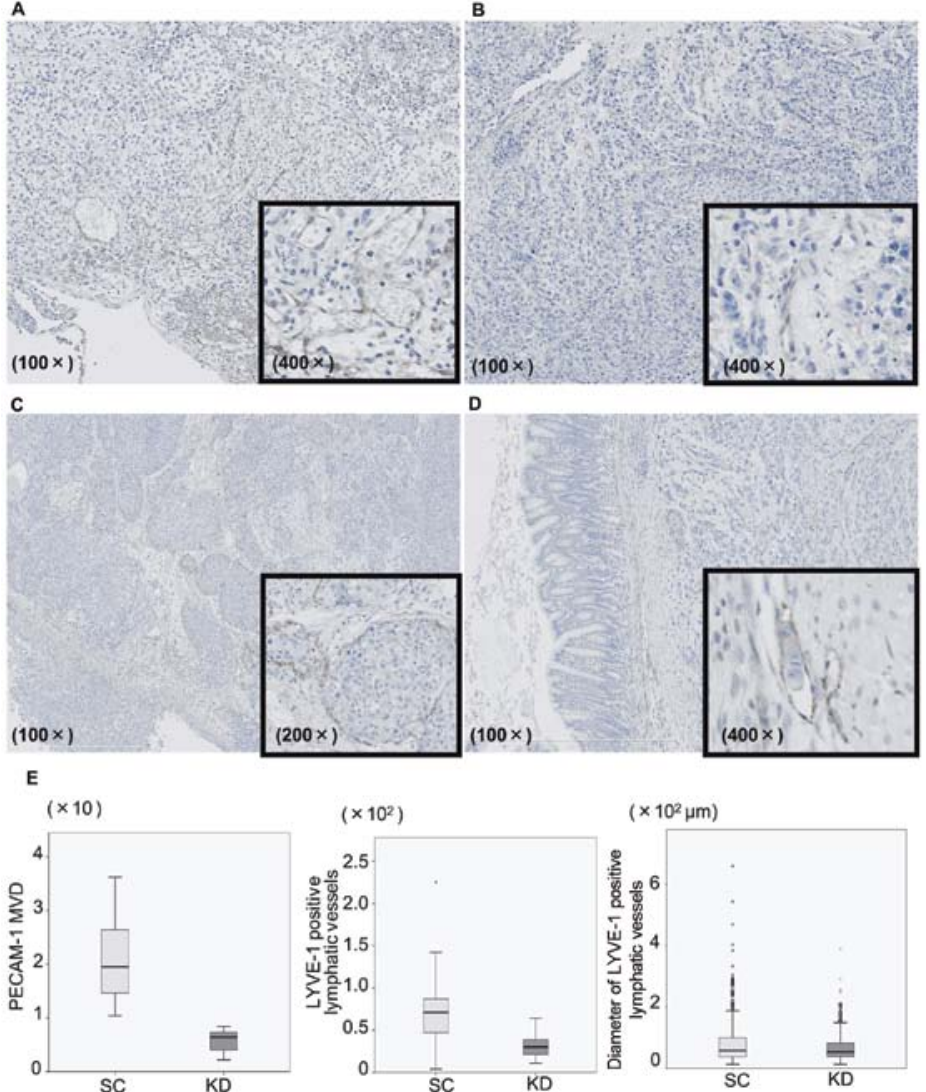

Figure 6. Assessment of the PECAM-1 and LYVE-1 expressions in the implanted stomach tumors in the o.i. mice. (A) Immunohistochemical staining of PECAM-1 in the SC-derived tumors. Vascular endothelial cells with PECAM-1-positive staining were present at the tumor periphery (x100). Blood vessels with a round shape were observed (x400). (B) Immunohistochemical staining of PECAM-1 in the KD-derived tumors. Vasculature formed by PECAM-1positive endothelial cells was found at the tumor periphery (x100). The vessel formation of the KD tumors was more poorly developed in both size and number compared with that of the SC tumors (x400). (C) Immunohistochemical staining of LYVE-1 in the SC-derived tumors. Lymphovascular endothelial cells with LYVE-1-positive staining were present at the tumor periphery (x100). Lymphatic vessels with cancer invasion were frequently observed (x400). (D) Immunohistochemical staining of LYVE-1 in the KD-derived tumors. The lymphatic vasculature in the KD tumors was more poorly developed in both size and number compared with that observed in the SC tumors (x400). (E) Left panel, the MVD estimated according to the presence of PECAM-1-positive vessels was significantly higher in the SC tumors than in the KD tumors $(\mathrm{P}=0.001)$. Middle panel, the number of lymphatic vessels with a positive LYVE-1 expression was significantly higher in the SC-derived tumors than in the KD-derived tumors $(\mathrm{P}=0.043)$. Right panel, the diameters of the lymphatic vessels with a positive LYVE-1 expression were significantly larger in the SC-derived tumors than in the KD-derived tumors $(\mathrm{P}=0.04)$.
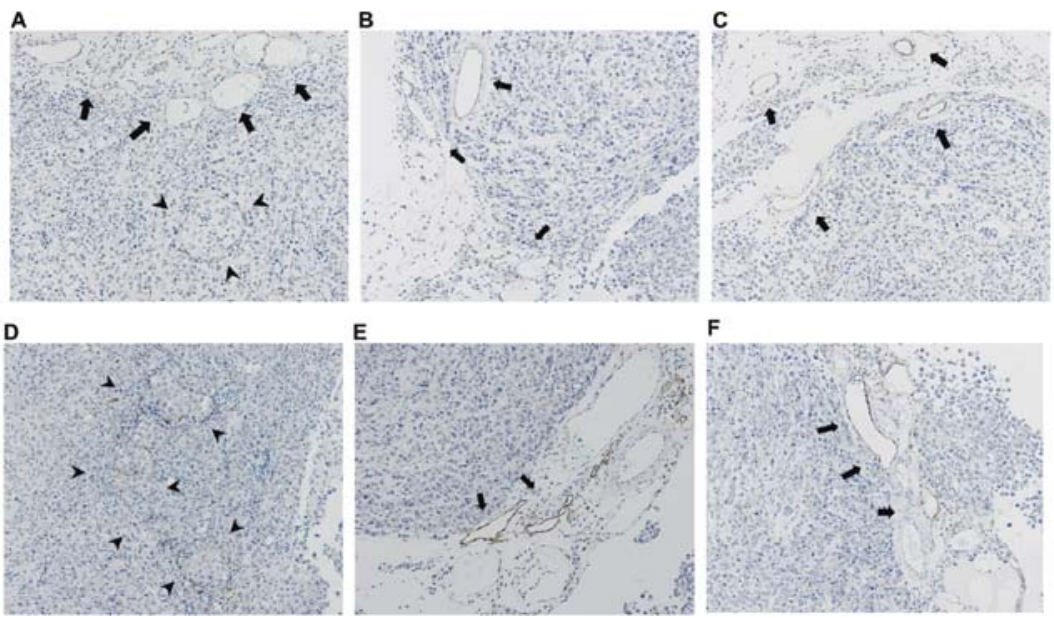

Figure 7. Microscopic features of the blood and lymphatic vessels in the disseminated nodules. (A) Immunohistochemical staining of PECAM-1 within the mesenteric nodules that formed in the SC o.i. mice. Blood vessels with a PECAM-1-positive expression developed at the tumor periphery (arrow). Vascular invasion in which the blood vessels contained cancer foci was also observed in the intratumoral regions (arrowhead). Immunohistochemical staining of PECAM-1 within the mesenteric nodules that formed in the SC i.p. (B) and KD i.p. mice (C). Blood vessels with a PECAM-1-positive expression developed at the tumor periphery in the SC i.p. and KD i.p. mice (arrow). However, no vessel formation was detected in the intratumoral regions in either type of mice. (D) Immunohistochemical staining of LYVE-1 within the mesenteric nodules that formed in the SC o.i. mice. Lymphatic invasion in which the lymphatic vessels contained cancer foci was frequently observed in the intratumoral regions (arrowhead). Immunohistochemical staining of LYVE-1 within the mesenteric nodules that formed in the SC i.p. (E) and KD i.p. mice (F). Lymphatic vessels with a LYVE-1-positive expression developed at the tumor periphery in the $\mathrm{SC}$ i.p. and KD i.p. mice (arrow). However, no lymphatic vessels were found in the intratumoral regions in either type of mice. 
These results indicate that the HIF-1 $\alpha$ expression may play an unfavorable role not only in the survival of free cancer cells in the peritoneal cavity, but also in the attachment of cells to the ECM of the mesentery. These in vitro data also support the in vivo findings that greater number of disseminated nodules formed in the KD i.p. mice. Based on the clear difference in the incidence of peritoneal dissemination between the o.i. and i.p. models, an alternative mechanism distinct from the seeding theory that acts on the development of peritoneal dissemination originating from primary tumors may exist.

Finally, the present study addressed the effects of HIF-1 $\alpha$ knockdown on tumor angiogenesis in o.i. and i.p. mice. The number as well as morphological features of the blood and lymphatic vessels in the tumors were immunohistochemically evaluated according to the PECAM-1 and LYVE-1 expression. In the stomach tumors formed by o.i., PECAM-1-positive endothelial cells grew at the periphery of the tumors derived from both the KD and SC cells. However, the MVD of the blood vessels was significantly higher in the SC tumors than in the KD tumors. These data strongly indicate that knockdown of the HIF-1 $\alpha$ expression attenuates angiogenesis within implanted tumors. Stoeltzing et al reported similar findings showing that suppression of HIF-1 $\alpha$ by the dominant negative form impairs angiogenesis and vessel maturation in stomach tumors. The authors concluded that inhibition of the HIF-1 $\alpha$ activity reduces VEGF secretion from cancer cells and leads to suppression of proangiogenic microenvironments in tumors (28). In the present study, hypoxic induction of the VEGF-A gene was suppressed by HIF-1 $\alpha$ knockdown. Therefore, the axis from HIF-1 $\alpha$ to VEGF-A may play an important role in the angiogenesis of SC-derived tumors. We further assessed the contribution of HIF-1 $\alpha$ to lymphangiogenesis using immunostaining of the lymphatic marker LYVE-1 in the SC and KD stomach tumors. The results showed that lymphatic vessels containing cancer cells frequently develop at the tumor periphery in both SC and KD tumors. However, the number as well as the diameter of the areas of lymphatic invasion were significantly greater in the SC tumors than in the KD tumors. These results indicate that the HIF-1 $\alpha$ expression accelerates lymphangiogenesis and lymphatic invasion in gastric tumors. Albrecht and Christofori recently reported that tumor cells under hypoxia secrete lymphangiogenic factors, such as VEGF-C, VEGF-D, PDGF-BB, Angiopoietin 1, Angiopoietin 2 and PIGF, all of which contribute to tumor lymphangiogenesis (29). We analyzed the hypoxic induction of VEGF-C and -D in an RT-PCR analysis; however, no expression of these factors was detected in the parental 58As9 cells (data not shown). Other secretory factors that accelerate lymphatic angiogenesis and lymphatic invasion may be induced by HIF-1 $\alpha$ in SC tumors. Taken together, the results suggest that HIF-1 $\alpha$ activates neovascularization of blood as well as lymphatic vessels and increases the intravasation of cancer cells within gastric tumors. These results further prompted us to hypothesize that the peritoneal dissemination observed in $\mathrm{SC}$ o.i. mice develops through an activated vascular network induced by HIF-1 $\alpha$. To assess this speculation, the presence of vascular formation in mesenteric nodules was investigated using SC i.p., KD i.p. and SC o.i. mice. Consequently, blood and lymphatic vessels without vascular invasion were observed at the tumor periphery in the nodules obtained from the SC i.p. and KD i.p mice. In contrast, vascular invasion, particularly in lymphatic vessels, was frequently observed in the intratumoral regions of the mesenteric nodules in the SC o.i. mice. This notable difference indicates that disseminated nodules in SC o.i. mice may be formed via the extravasation of cancer cells. Moreover, cancer cells present on the mesentery may be primarily interconnected to stomach tumors through vascular networks. Silvermann reported that the subperitoneal space consists of fatty tissue, blood vessels, lymphatics and lymph nodes enveloped by a serosal lining. This space provides a complex interconnecting network that is an important conduit for tumor cells within the peritoneal cavity (30). This report strongly supports our rationale that peritoneal dissemination is a type of distant metastasis that travels through a transvessel route whereby HIF-1 $\alpha$ initiates tumor angiogenesis within the primary tumor.

In conclusion, the present study demonstrated for the first time that HIF-1 $\alpha$ is a crucial factor involved in the development of peritoneal dissemination via the natural metastatic route in scirrhous gastric cancer. Our report also provides a possible mechanism, breaking through the conventional concept that HIF-1 $\alpha$ activates tumor angiogenesis, showing that HIF-1 $\alpha$ may increase metastasis to the peritoneum via vascular networks. However, in order to elucidate this novel mechanism, it must be clarified whether inhibition of vascular formation leads to the suppression of peritoneal dissemination in the 58As 9 o.i. model. In the future, the clinical use of HIF-1 $\alpha$ inhibitors is expected to suppress peritoneal dissemination of scirrhous gastric cancer by impairing tumor angiogenesis.

\section{Acknowledgements}

We would like to thank Mr. F. Mutoh for his valuable contributions to the immunohistochemical studies.

\section{References}

1. Garcia M, Jemal A, Ward EM, Center MM, Hao Y, Siegel RL and Thun MJ: Global Cancer Facts and Figures 2007. American Cancer Society, Atlanta, GA, 2007.

2. Chu DZ, Lang NP, Thompson C, Osteen PK and Westbrook KC: Peritoneal carcinomatosis in non gynecologic malignancy. A prospective study of prognostic factors. Cancer 63: 364-367, 1989.

3. Yonemura Y, Kawamura T, Bandou E, Tsukiyama G, Endou Y and Miura M: The natural history of free cancer cells in the peritoneal cavity. In: Advances in Peritoneal Surface Oncology. Gonzalez-Moreno S (ed). Springer, Berlin, pp11-23, 2007.

4. Kotanagi H, Saito Y, Shiozawa N and Koyama K: Establishment of a human cancer cell line with high potential for peritoneal dissemination. J Gastroenterol 30: 437-438, 1995.

5. Yanagihara K, Takigahira M, Tanaka H, et al: Development and biological analysis of peritoneal metastasis mouse models for human scirrhous stomach cancer. Cancer Sci 96: 323-332, 2005.

6. Takei Y, Takigahira M, Mihara K, Tarumi Y and Yanagihara $\mathrm{K}$ : The metastasis-associated microRNA miR-516a-3p is a novel therapeutic target for inhibiting peritoneal dissemination of human scirrhous gastric cancer. Cancer Res 71: 1442-1453, 2010.

7. Wang GL and Semenza GL: General involvement of hypoxiainducible factor 1 in transcriptional response to hypoxia. Proc Natl Acad Sci USA 90: 4304-4308, 1993.

8. Semenza GL: HIF-1 and tumor progression: pathophysiology and therapeutics. Trends Mol Med 8: S62-7, 2002.

9. Semenza GL: Targeting HIF-1 for cancer therapy. Nat Rev Cancer 3: 721-732, 2003.

10. Kitajima Y and Miyazaki K: The critical impact of HIF-1 $\alpha$ on gastric cancer biology. Cancers 5: 15-26, 2013. 
11. Sumiyoshi Y, Kakeji Y, Egashira A, Mizokami K, Orita H and Maehara Y: Overexpression of hypoxia-inducible factor $1 \alpha$ and p53 is a marker for an unfavorable prognosis in gastric cancer. Clin Cancer Res 12: 5112-5117, 2006.

12. Koukourakis MI, Giatromanolaki A, Skarlatos J, et al: Hypoxia inducible factor (HIF-1 $\alpha$ and HIF-2 $\alpha$ ) expression in early esophageal cancer and response to photodynamic therapy and radiotherapy. Cancer Res 61: 1830-1832, 2001.

13. Bos R, van der Groep P, Greijer AE, et al: Levels of hypoxiainducible factor- $1 \alpha$ independently predict prognosis in patients with lymph node negative breast carcinoma. Cancer 97: 1573-1581, 2003.

14. Nakanishi K, Hiroi S, Tominaga S, et al: Expression of hypoxiainducible factor-1 $\alpha$ protein predicts survival in patients with tranitional cell carcinoma of the upper urinary tract. Clin Cancer Res 11: 2583-2590, 2005.

15. Birner P, Schindl M, Obermair A, Breitenecker G and Oberhuber G: Expression of hypoxia-inducible factor $1 \alpha$ in epithelial ovarian tumors: its impact on prognosis and on response to chemotherapy. Clin Cancer Res 7: 1661-1668,2001.

16. Nakamura J, Kitajima Y, Kai K, Hashiguchi K, Hiraki M, Noshiro $\mathrm{H}$ and Miyazaki K: HIF-lalpha is an unfavorable determinant of relapse in gastric cancer patients who underwent curative surgery followed by adjuvant 5-FU chemotherapy. Int J Cancer 127: 1158-1171, 2010.

17. Ide T, Kitajima Y, Miyoshi A, et al: Tumor-stromal cell interaction under hypoxia increases the invasiveness of pancreatic cancer cells through the hepatocyte growth factor/c-Met pathway. Int J Cancer 119: 2750-2759, 2006.

18. Kitajima Y, Ide T, Ohtsuka T and Miyazaki K: Induction of hepatocyte growth factor activator gene expression under hypoxia activates the hepatocyte growth factor/c-Met system via hypoxia inducible factor-1 in pancreatic cancer. Cancer Sci 99: 1341-1347, 2008.

19. Liu L, Sun L, Zhang H, et al: Hypoxia-mediated up-regulation of MGrl-Ag/37LRP in gastric cancers occurs via hypoxiainducible-factor 1-dependent mechanism and contributes to drug resistance. Int J Cancer 124: 1707-1715, 2009.
20. Song IS, Wang AG, Yoon SY, Kim JM, Kim JH, Lee DS and Kim NS: Regulation of glucose metabolism-related genes and VEGF by HIF- $1 \alpha$ and HIF-1 $\beta$, but not HIF- $2 \alpha$, in gastric cancer. Exp Mol Med 41: 51-58, 2009.

21. Zhang R,Fu H, Chen D, Hua J,Hu Y,Sun K and Sun X: Subcellular distribution of S100A4 and its transcriptional regulation under hypoxic conditions in gastric cancer cell line BGC823. Cancer Sci 101: 1141-1146, 2010.

22. Liu L, Ning X, Sun L, et al: Hypoxia-inducible factor-1a contributes to hypoxia-induced chemoresistance in gastric cancer. Cancer Sci 99: 121-128, 2008.

23. Miyoshi A, Kitajima Y, Ide T, et al: Hypoxia accelerates cancer invasion of hepatoma cells by upregulating MMP expression in an HIF-1 $\alpha$-independent manner. Int J Oncol 29: 1533-1539, 2006.

24. Xin L and Yibin K: Hypoxia and hypoxia-inducible factors: master regulators of metastasis. Clin Cancer Res 16: 5928-5935, 2010.

25. Hiraki M, Kitajima Y, Kai K, Nakamura J, Hashiguchi K, Noshiro $\mathrm{H}$ and Miyazaki K: Knockdown of hypoxia-inducible factor-1a accelerates peritoneal dissemination via the upregulation of MMP-1 expression in gastric cancer cell lines. Exp Ther Med 4: 355-362, 2012.

26. Liotta LY: Tumor invasion and metastases - role of the extracellular matirix: Rhoads Memorial Award lecture. Cancer Res 46: 1-7, 1986.

27. Kawamura T, Endo Y, Yonemura Y, et al: Significance of integrin alpha2/beta1 in peritoneal dissemination of a human gastric cancer xenograft model. Int J Oncol 18: 809-815, 2001.

28. Stoeltzing O, McCarty MF, Wey JS, et al: Role of hypoxiainducible factor 1alpha in gastric cancer cell growth, angiogenesis and vessel maturation. J Natl Cancer Inst 96: 946-956, 2004.

29. Albrecht I and Christofori G: Molecular mechanisms of lymphangiogenesis in development and cancer. Int J Dev Biol 55: 483-494, 2011.

30. Silverman MP: The subperitoneal space: mechanisms of tumor spread in the peritoneal cavity, mesentery and omentum. Cancer Imaging 4: 25-29, 2003. 